\section{Pleuropulmonary infection with chest wall infiltration by Eikenella corrodens}

\author{
J W W Killen, G L Swift, R J White
}

\begin{abstract}
Eikenella corrodens is a facultative anaerobic bacillus which is part of the normal flora of the oral cavity and has an unusual antibiotic sensitivity for an anaerobe. The case history is presented of a young man with chest wall infiltration by Eikenella corrodens.
\end{abstract}

(Thorax 1996;51:871-872)

Keywords: Eikenella corrodens, pleural infection, pulmonary infection.

A 33 year old man presented with a swelling of the left chest wall. Three months previously he had had a febrile illness followed by left chest pain which lasted for one week. He described the pain as an ache, increasing in intensity and becoming pleuritic. This was diagnosed as a chest infection on the basis of a leucocytosis, elevated plasma viscosity, and a chest radiograph showing consolidation at the left base, though there was never any sputum production. No further investigations were done at that time and he was treated with amoxycillin $500 \mathrm{mg}$ three times daily for five days. The symptoms settled for two months but he then complained of a tender lump on the left anterior chest wall. On direct questioning he admitted to weight loss of $15 \mathrm{~kg}$ in two months and night sweats for two weeks. He smoked 20 cigarettes daily. Before this episode his health had been excellent, and he denied activity related to viral immunodeficiency. On examination he had a hard tender erythematous swelling on the left chest wall anteriorly, just below the nipple, measuring approximately $3 \times 5 \mathrm{~cm}$

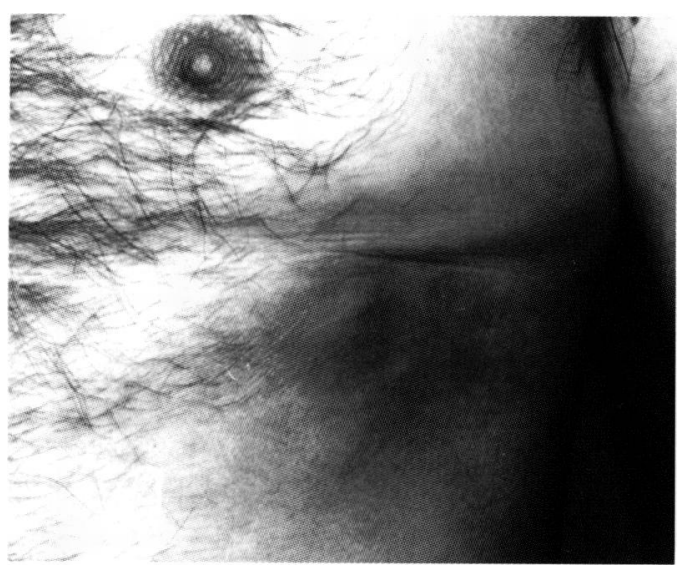

Figure 1 Anterior view of left chest showing the inflammatory swelling inferior to the left nipple. (fig 1), and he was tender in the left upper quadrant of the abdomen. Examination of the buccal cavity revealed nothing remarkable.

A repeat chest radiograph still showed some left basal consolidation and the white cell count was still raised at $14.6 \times 10^{9}$, of which $70 \%$ were neutrophils and $10 \%$ were monocytes. The plasma viscosity was markedly raised at 2.37 (normal range $1.5-1.72 \mathrm{cP}$ ). A computed tomographic scan of the thorax (fig 2) showed patchy areas of dense air space consolidation in the lingula segments, the anterior segments of the left upper lobe, and the posterior segment of the left lower lobe. There was moderate pleural thickening associated with these areas, and anterolaterally a soft tissue mass extended through the chest wall externally into the subcutaneous tissues, centrally to involve the pericardiac fat, pleura and pleural space, and through the diaphragm into the peritoneal fat anterior to the spleen and stomach. The pre-aortic lymph nodes were enlarged.

Needle aspiration revealed a small quantity of pus which yielded scanty Gram positive cocci on microscopy, but no significant culture was reported after five days. A Tru-cut biopsy specimen comprised dense fibrous connective tissue with focal areas of inflammatory cellular infiltrate. No sulphur granules were seen. On the basis of the Gram stain he was started on penicillin $\mathrm{V}$ and flucloxacillin and discharged. Two weeks later a further lump had appeared, medial to the left nipple, but the erythema and splenic tenderness had resolved. Antibiotics were changed to co-amoxyclav, but after a further two weeks another swelling had appeared, lateral to the original site. A computed tomographic scan showed progression in some areas and partial regression in others. Antibiotics were stopped and an open biopsy specimen was taken. Histological examination was once again uninformative but, after five days, there was a heavy growth of Eikenella corrodens which was sensitive to the ampicillin group of antibiotics, cephradine, and cefuroxime. $\mathrm{He}$ was subsequently given a prolonged course of oral amoxycillin, $500 \mathrm{mg}$ three times daily.

After six weeks of antibiotics his infection had resolved clinically but he was left with a small sinus at the site of the later biopsy. Six months later he had another episode of pain

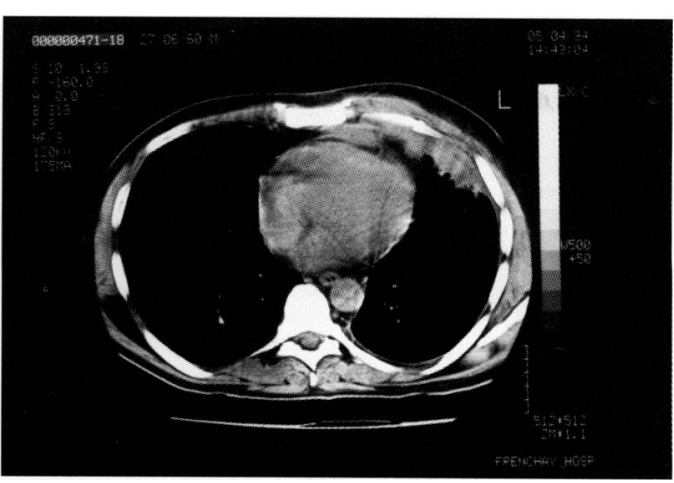

Figure 2 High resolution computed tomographic section $1.5 \mathrm{~mm}$ thick (mediastinal window) showing left pleural thickening abutting the pericardium with infiltration through the anterior chest wall into the subcutaneous tissues. 
and erythema in the same area of the chest wall which again responded to amoxycillin. There has been no growth on repeated swabs of the sinus. He has now started on prophylactic amoxycillin $250 \mathrm{mg}$ twice daily.

\section{Discussion}

Eikenella corrodens is a Gram negative bacillus and facultative anaerobe. Although it was first described in $1948,{ }^{1}$ it has only recently been found to be pathogenic. It is part of the normal flora of the oral cavity, the gastrointestinal tract, and the genitourinary tract. Clinical infection is unusual, and most cases are associated with periodontal disease or bite wounds. Deep seated infections, including endocarditis, osteomyelitis, arthritis, pelvic infections, sinusitis, parotitis, cellulitis, central nervous system infection, and chorioamnionitis have been reported, often with abscess formation. ${ }^{2}$ Though pure isolates from infected material do occur, most cases are found to have mixed infections. A pure growth of Eikenella corrodens was obtained in our case. This is unlikely to be secondary to his previous antibiotic therapy because the material from the first aspiration and biopsy specimen was cultured under standard conditions, prior to antibiotic therapy, and no growth was obtained. The possibility of synergism between Eikenella corrodens and streptococcal strains has been raised, particularly in the formation of abscesses. ${ }^{3}$

A series of 24 cases of pleuropulmonary infection due to Eikenella corrodens has been reviewed by Joshi et $a l,{ }^{4}$ of which 15 had pleural effusion, one had pleural thickening, and none had infiltration of adjoining structures. Most $(75 \%)$ of the patients in this series had some pre-existing disease and $33 \%$ had an underlying malignancy. Zgheib et al ${ }^{5}$ reported a case of mediastinitis, pericardial and pleural effusion, and bronchopneumonia following peritonsillar abscess drainage, without preexisting disease, in which Eikenella corrodens was grown from the blood cultures. This case was treated with intravenous ticarcillin $5 \mathrm{~g}$ four times daily for three weeks and pleural and pericardial drainage. Both of these papers and others emphasise the unusual antibiotic susceptibility pattern of this anaerobe, which is almost always sensitive to ampicillin and resistant to clindamycin, antistaphylococcal penicillins, aminoglycosides, erythromycin, and metronidazole. Susceptibility to penicillin $\mathrm{G}$ and cephalosporins varies.

The case we have presented, with infection of the lung pleura and chest wall, may be unique as infiltration of the chest wall has not been previously reported. Although not fully excluded, there was no evidence of predisposing factors such as occult malignancy or immunodeficiency, or causes of antecedent bacteraemia such as poor dentition or oral surgery. Initial antibiotics were unsuccessful because of resistance or inadequate length of treatment. This case therefore emphasises the pathogenic potential of Eikenella corrodens and the difficulties of treating infections with this organism.

1 Henriksen SD. Studies in gram-negative anaerobes. II. Gram-negative anaerobic rods with spreading colonies. Acta Pathol Microbiol Scand 1948;25:368-75.

2 Danziger LH, Schoonover LL, Kale P, Resnick DJ. Eikenella corrodens as an intra-abdominal pathogen. Am Surg 1994; 60:296-9.

3 Stone DR. Eikenella corrodens and group C streptococci (letter). Clin Infect Dis 1992;14:789.

4 Joshi N, O'Bryan T, Appelbaum P. Pleuropulmonary infections caused by Eikenella corrodens. Clin Infect Dis 1991;13:1207-12.

5 Zgheib A, el Allaf D, Demonty J, Rorive G. Intrathoracic infections with bacteraemia due to Eikenella corrodens as a complication of peritonsillar abscesses: report of a case and review of the literature. Acta Clin Belg 1992;47:124-8. 